PHYSICAL REVIEW D 90, 101701(R) (2014)

\title{
Superintegrability of generalized Calogero models with oscillator or Coulomb potential
}

\author{
Tigran Hakobyan \\ Yerevan State University, 1 Alex Manoogian Street, Yerevan 0025, Armenia \\ Olaf Lechtenfeld ${ }^{\dagger}$ \\ Leibniz Universität Hannover, Appelstraße 2, 30167 Hannover, Germany
}

Armen Nersessian

Yerevan State University, 1 Alex Manoogian Street, Yerevan 0025, Armenia and Tomsk Polytechnic

University, Lenin Avenue 30, 634050 Tomsk, Russia

(Received 14 October 2014; published 25 November 2014)

\begin{abstract}
We deform $N$-dimensional (Euclidean, spherical and hyperbolic) oscillator and Coulomb systems, replacing their angular degrees of freedom by those of a generalized rational Calogero model. Using the action-angle description, it is established that maximal superintegrability is retained. For the rational Calogero model with Coulomb potential, we present all constants of motion via matrix model reduction. In particular, we construct the analog of the Runge-Lenz vector.
\end{abstract}

DOI: $10.1103 /$ PhysRevD.90.101701

PACS numbers: 03.65.-w, 02.30.Ik

\section{INTRODUCTION}

The rational Calogero model [1] and its various generalizations, based on arbitrary Coxeter root systems [2] or with trigonometric and elliptic potentials [3]), continue to attract interest. The reason is their rich integrability structure and their widespread applications (for a review, see Ref. [4]). One of the striking features of rational $N$-particle $\left(A_{N-1}\right)$ Calogero models is their maximal superintegrability (i.e. existence of $2 N-1$ functionally independent constants of motion), first established by Wojcechowski [5]. This property is retained for other root systems, and it admits the addition of an external oscillator potential [6], which we call the Calogero-oscillator system. Superintegrability has been established also for the hyperbolic Calogero model [7] and for the relativistic version $[8,9]$ known as the rational Ruijsenaars-Schneider model [10].

One may ask whether other potentials (different from the oscillator one) may be added to the rational Calogero model without destroying its integrability. To answer this question, it is fruitful to reinterpret the model as describing a single nonrelativistic particle moving in $\mathbb{R}^{N}$ in the presence of a particular potential given by the root system (e.g. $\left.A_{N-1}\right)$. The conformal invariance of this system then suggests passing to spherical coordinates $\left(r, \theta_{i}\right)$ with $i=1, \ldots, N-1$ and trying to add a "radial" potential $V(r)$. As an important example, Khare [11] (see also

\footnotetext{
*tigran.hakobyan@ysu.am †lechtenf@itp.uni-hannover.de *arnerses@ysu.am
}

Ref. [12]) proposed an exactly solvable rational Calogero model with a Coulomb-like radial potential, to which we will refer as the Calogero-Coulomb model. This was followed by a remark of Calogero [13] that any radial deformation of the Calogero model by a rotationally invariant exactly solvable potential remains exactly solvable.

In this paper we establish maximal superintegrability for the Calogero-Coulomb and Calogero-oscillator models associated to any Coxeter root system, as well as for their generalizations from $\mathbb{R}^{N}$ to the $N$-sphere $S^{N}$ and the (twosheeted) hyperboloid $H^{N}$. Furthermore, we suggest that these Calogero-type deformations of the $N$-dimensional oscillator and Coulomb systems are their only modifications preserving maximal superintegrability.

The construction of these models proceeds in two steps. First, we rewrite the $N$-dimensional oscillator or Coulomb system in spherical coordinates, and second we replace the angular part of either system [given by the quadratic $S O(N)$ Casimir] with the angular part of a rational Calogero model [given by its $S L(2, \mathbb{R})$ Casimir], which has recently been considered in a series of papers [14-18]. A proof of maximal superintegrability directly follows from the formulation of these systems in terms of action-angle variables [16]. The latter requires the knowledge of an action for the angular part of the rational Calogero model, which we get by taking the classical limit of its energy spectrum, as constructed in Ref. [18].

As an example, we consider the rational $A_{N-1}$ Calogero model with a Coulomb potential, for which we present all classical constants of motion via the well known matrix-model reduction procedure [2,19]. In particular, we determine the analog of the Runge-Lenz vector. The quantum integrals are briefly discussed using the Dunkl operator approach [20]. 


\section{ACTION-ANGLE VARIABLES AND SUPERINTEGRABILITY}

The description of an integrable system in terms of its action-angle variables provides a convenient way to determine the hidden symmetries. Concretely, consider an $N$-dimensional system with action-angle variables $\left(I_{i} \in\right.$ $\left.\mathbb{R}, \Phi_{j} \in[0,2 \pi)\right)$ for $i, j=1, \ldots, N$ and a Hamiltonian of the form

$$
\mathcal{H}=\mathcal{H}\left(n I_{1}+m I_{2}, I_{3}, \ldots, I_{N}\right) \text { with }\left\{I_{i}, \Phi_{j}\right\}=\delta_{i j},
$$

where $n$ and $m$ are integers. This system features a hidden symmetry, given by the additional constant of motion

$$
I_{\text {hidden }}=A \cos \left(m \Phi_{1}-n \Phi_{2}+\alpha\right),
$$

with $A(I)$ and $\alpha(I)$ being arbitrary functions of the action variables. By iteration, the Hamiltonian of a maximally superintegrable system should depend only on one integer linear combination of the action variables,

$$
\mathcal{H}=\mathcal{H}\left(n_{1} I_{1}+m_{2} I_{2}+\ldots n_{N} I_{N}\right),
$$

where $n_{1}, \ldots, n_{N}$ are integers. In that case the $\frac{1}{2} N(N-1)$ functions

$$
I_{i j}=A_{i j}(I) \cos \left(n_{j} \Phi_{i}-n_{i} \Phi_{j}+\alpha_{i j}(I)\right)
$$

all are constants of motion functionally independent from the Liouville integrals $I_{1}, \ldots, I_{N}$. From this set we can choose $N-1$ functionally independent ones, say $I_{i, i+1}$ with $i=1, \ldots N-1$. Hence, such a system possesses $2 N-1$ functionally independent constants of motion; i.e. it is maximally superintegrable.

In a recent paper [16] we have constructed integrable deformations of $\mathrm{N}$-dimensional oscillator and Coulomb systems on Euclidean spaces, spheres and (two-sheeted) hyperboloids, by replacing their "angular part" (on $S^{N-1}$ ) by an arbitrary $(N-1)$-dimensional integrable system, formulated in terms of action-angle variables. On the Euclidean space $\mathbb{R}^{N}$, these deformations are defined by

$$
\mathcal{H}=\frac{p_{r}^{2}}{2}+\frac{\mathcal{I}\left(I_{i}\right)}{r^{2}}+V(r) \quad \text { with } \quad\left\{p_{r}, r\right\}=1,
$$

where the radial potential $V(r)$ could be either

$$
V_{\mathrm{osc}}(r)=\frac{\omega^{2} r^{2}}{2} \quad \text { or } \quad V_{\mathrm{Coul}}(r)=-\frac{\gamma}{r}
$$

and $\mathcal{I}\left(I_{i}\right)$ is the Hamiltonian of some compact $(N-1)$ dimensional integrable system formulated in action-angle variables $\left(I_{i}, \Phi_{i}\right)$ with $i=1, \ldots, N-1$.

The analogous systems on the $N$-sphere $S^{N}$ and the (two-sheet) hyperboloid $H^{N}$ of size $r_{0}$ are

$$
\begin{aligned}
& S^{N}: \mathcal{H}=\frac{p_{\chi}^{2}}{2 r_{0}^{2}}+\frac{\mathcal{I}}{r_{0}^{2} \sin ^{2} \chi}+V(\tan \chi), \quad V_{\mathrm{Higgs}}=\frac{r_{0}^{2} \omega^{2}}{2} \tan ^{2} \chi, \quad V_{\mathrm{Schr}}=-\frac{\gamma}{r_{0}} \cot \chi, \\
& H^{N}: \mathcal{H}=\frac{p_{\chi}^{2}}{2 r_{0}^{2}}+\frac{\mathcal{I}}{r_{0}^{2} \sinh ^{2} \chi}+V(\tanh \chi), \quad V_{\mathrm{Higgs}}=\frac{r_{0}^{2} \omega^{2}}{2} \tanh ^{2} \chi, \quad V_{\mathrm{Schr}}=-\frac{\gamma}{r_{0}} \operatorname{coth} \chi,
\end{aligned}
$$

with $\left\{p_{\chi}, \chi\right\}=1$ and $\mathcal{I}\left(I_{i}\right)$ depending on the respective action variables. The oscillator and Coulomb potentials $V_{\text {higgs }}$ and $V_{\text {schr }}$ on spheres and hyperboloids have been proposed by Higgs [21] and by Schrödinger [22]), respectively. In other words, we obtain integrable deformations of the $N$-dimensional oscillator and Coulomb systems by replacing the quadratic $S O(N)$ Casimir invariant with the Hamiltonian $\mathcal{I}$ of some $(N-1)$-dimensional compact integrable system.

It was shown [16] that the Hamiltonians of the oscillatorlike systems are given by

$$
\mathcal{H}_{\mathrm{osc}}=\mathcal{H}_{\mathrm{osc}}\left(2 I_{r}+\sqrt{2 \mathcal{I}}\right)= \begin{cases}\omega\left(2 I_{r}+\sqrt{2 \mathcal{I}}\right) & \text { for } \mathbb{R}^{N}, \\ \frac{1}{2}\left(2 I_{\chi}+\sqrt{2 \mathcal{I}}+\omega\right)^{2}-\frac{\omega^{2}}{2} & \text { for } S^{N}, \\ -\frac{1}{2}\left(2 I_{\chi}+\sqrt{2 \mathcal{I}}-\omega\right)^{2}+\frac{\omega^{2}}{2} & \text { for } H^{N} .\end{cases}
$$

Similarly, the Hamiltonians of the Coulomb-like systems read

$$
\mathcal{H}_{\text {Coul }}=\mathcal{H}_{\text {Coul }}\left(I_{r}+\sqrt{2 \mathcal{I}}\right)= \begin{cases}-\frac{1}{2} \gamma^{2}\left(I_{r}+\sqrt{2 \mathcal{I}}\right)^{2} & \text { for } \mathbb{R}^{N}, \\ -\frac{1}{2} \gamma^{2}\left(I_{\chi}+\sqrt{2 \mathcal{I}}\right)^{2}+\frac{1}{2}\left(I_{\chi}+\sqrt{2 \mathcal{I}}\right)^{2} & \text { for } S^{N}, \\ -\frac{1}{2} \gamma^{2}\left(I_{\chi}+\sqrt{2 \mathcal{I}}\right)^{2}-\frac{1}{2}\left(I_{\chi}+\sqrt{2 \mathcal{I}}\right)^{2} & \text { for } H^{N}\end{cases}
$$

Thus, if the angular Hamiltonian has the form

$$
\mathcal{I}=\frac{1}{2}\left(\sum_{i} k_{i} I_{i}+\text { const }\right)^{2} \quad \text { with } \quad k_{i} \in \mathbb{Z}
$$

then the respective deformations of the oscillator and Coulomb systems are maximally superintegrable. The deformed systems will have the same configuration space as initial one if $\mathcal{I}$ is a system on the $(N-1)$-sphere. 
The key observation in this paper is that any compact system with Hamiltonian (11) is precisely the angular part of a rational Calogero model, which has been suggested and studied in Refs. [14,15,18].

\section{CALOGERO-COULOMB AND CALOGERO-OSCILLATOR MODELS}

The quantum Hamiltonian of the generalized rational Calogero model associated with a Coxeter root system

$\{\alpha\}=\left\{\left(\alpha_{1}, \alpha_{2}, \ldots, \alpha_{N}\right)\right\}=\mathcal{R} \subset \mathbb{R}^{N} \ni \quad\left(x_{1}, x_{2}, \ldots, x_{N}\right) \equiv x$

reads [2]

$$
\begin{aligned}
& \hat{\mathcal{H}}_{\text {Cal }}=\sum_{i} \frac{\hat{p}_{i}^{2}}{2}+\sum_{\alpha \in \mathcal{R}_{+}} \frac{g_{\alpha}\left(g_{\alpha}-\hbar\right)(\alpha \cdot \alpha)}{2(\alpha \cdot x)^{2}} \\
& \text { where }\left[\hat{p}_{i}, x_{j}\right]=-\mathrm{i} \hbar \delta_{i j},
\end{aligned}
$$

$\mathcal{R}_{+}$is the subset of positive roots, and $g_{\alpha} \geq 0$ is a multiplicity function on $\mathcal{R}$ invariant under the Weyl reflection group $W$ [23]. We indicate quantum objects by "hatting" them. The Hamiltonian $\hat{\mathcal{I}}$ of the angular part of $\hat{\mathcal{H}}_{\text {cal }}$ is defined by rewriting the latter in spherical variables,

$\hat{\mathcal{H}}_{\text {Cal }}=\frac{\hat{p}_{r}^{2}}{2}+\frac{\hat{\mathcal{I}}}{r^{2}}+\frac{\hbar^{2}(N-1)(N-3)}{8 r^{2}} \quad$ with $r^{2}=\sum_{i} x_{i}^{2}$

and $\hat{p}_{r}=-\mathrm{i} \hbar \partial_{r}$.

The basic classical properties of $\hat{\mathcal{I}}$ were considered in Refs. [14-16], and its quantum features were investigated in Ref. [18]. There, the energy spectrum was found to be

$$
\begin{aligned}
\mathcal{E}_{k_{2}, k_{3}, k_{4}, \ldots} & =\frac{1}{2} q(q+\hbar(N-2)) \quad \text { with } \\
q & =\sum_{\alpha \in \mathcal{R}_{+}} g_{\alpha}+\hbar \sum_{i=2}^{N} d_{i} k_{i}, \quad \text { and } \quad k_{i}=0,1,2, \ldots
\end{aligned}
$$

Here, $d_{1}=2, d_{2}, \ldots, d_{N}$ are the degrees of the basic homogeneous $W$-invariant polynomials $\sigma_{1}=r^{2}, \sigma_{2}, \ldots, \sigma_{N}$. Note that the quantum number $k_{1}$ from the contribution of $\sigma_{1}$ is absent in the energy formula.
Since in the classical limit $\hbar \rightarrow 0$ the quantum numbers $\hbar k_{i}$ reduce to the action variables $I_{i}$, the above relation yields the classical Hamiltonian in terms of action variables:

$$
\mathcal{I}=\lim _{\hbar \rightarrow 0} \frac{1}{2} q^{2}=\frac{1}{2}\left(\sum_{i=2}^{N} d_{i} I_{i}+\sum_{\alpha \in \mathcal{R}_{+}} g_{\alpha}\right)^{2} .
$$

In particular, the angular part of rational $A_{N-1}$ Calogero model is given by

$$
\mathcal{I}=\frac{1}{2}\left(\frac{N(N-1)}{2} g+I_{1}+3 I_{3}+\cdots+N I_{N}\right)^{2} .
$$

Note that, compared to (16), the index $i$ has been shifted up by 1 , and there is an additional contribution of $I_{1}$ from the center of mass which adds an extra $A_{1}$ to the $A_{N-1}$ root system. We see that the angular part of the generalized rational Calogero model belongs to the class (11); i.e. it is suitable for the construction of superintegrable deformations of oscillator and Coulomb systems.

Substituting (17) into (5)-(8), we obtain classical maximally superintegrable deformations of (Euclidean, spherical and pseudospherical) oscillator and Coulomb systems. In Euclidean space $\mathbb{R}^{N}$, these systems are then characterized by the Hamiltonians

$$
\begin{aligned}
\mathcal{H}_{\text {osc }} & =\frac{p^{2}}{2}+\sum_{\alpha \in \mathcal{R}_{+}} \frac{g_{\alpha}^{2}(\alpha \cdot \alpha)}{2(\alpha \cdot x)^{2}}+\frac{\omega^{2} x^{2}}{2} \text { and } \\
\mathcal{H}_{\text {Coul }} & =\frac{p^{2}}{2}+\sum_{\alpha \in \mathcal{R}_{+}} \frac{g_{\alpha}^{2}(\alpha \cdot \alpha)}{2(\alpha \cdot x)^{2}}-\frac{\gamma}{x},
\end{aligned}
$$

where $p \equiv\left(p_{1}, p_{2}, \ldots, p_{N}\right)$ and $x \equiv\left(x_{1}, x_{2}, \ldots, x_{N}\right)$. The maximal superintegrability is well known for the oscillator case, due to the knowledge of oscillating quantities with integer frequencies in the harmonic potential $[2,6]$. The Calogero-Coulomb system seems much less known $[11,13],{ }^{1}$ and its superintegrability has not been discussed in the literature yet. Here we have established that the generalized rational Calogero-Coulomb model for any Coxeter root system is maximally superintegrable.

The story extends from Euclidean space to spaces of constant curvature, i.e. to the $\mathrm{N}$-dimensional sphere and pseudosphere, which we present in terms of Euclidean coordinates on the ambient space $\mathbb{R}^{N+1} \ni\left(x_{0}, x\right)$,

$$
\begin{aligned}
& \mathcal{H}_{\text {Coul }}^{(p) s}=\frac{p^{2}}{2} \mp \frac{(x \cdot p)^{2}}{2 r_{0}^{2}}+\sum_{\alpha \in \mathcal{R}_{+}} \frac{g_{\alpha}^{2}(\alpha \cdot \alpha)}{2(\alpha \cdot x)^{2}}-\frac{\gamma}{r_{0}} \frac{x_{0}}{x} \quad \text { with } \quad x_{0}^{2} \pm x^{2}=r_{0}^{2}, \\
& \mathcal{H}_{\mathrm{osc}}^{(p) s}=\frac{p^{2}}{2} \mp \frac{(x \cdot p)^{2}}{2 r_{0}^{2}}+\sum_{\alpha \in \mathcal{R}_{+}} \frac{g_{\alpha}^{2}(\alpha \cdot \alpha)}{2(\alpha \cdot x)^{2}}+\frac{\omega^{2} r_{0}^{2}}{2} \frac{x^{2}}{x_{0}^{2}} \quad \text { with } \quad x_{0}^{2} \pm x^{2}=r_{0}^{2} .
\end{aligned}
$$

\footnotetext{
${ }^{1}$ In these papers only the rational $A_{N-1}$ Calogero model was considered. However, the generalization to arbitrary Coxeter root systems is straightforward.
} 
In these expressions, the upper sign corresponds to the sphere and lower sign to the hyperboloid.

As in the Euclidean case, these systems are superintegrable. Their potentials may be viewed as a higher-dimensional generalizations of superintegrable deformations of twodimensional oscillator and Coulomb potentials which are known as Tremblay-Turbiner-Winternitz [24] and PostWinternitz [25] systems, respectively. Generalizations of the latter to spheres and pseudospheres have been proposed in Ref. [16]. We note that the two-dimensional Post-Winternitz system possesses just three constants of motion, which are all quadratic in the momenta. This fact implies that it admits a separation of variables in some coordinate system different from the spherical one. Since the Tremblay-TurbinerWinternitz and Post-Winternitz systems are connected by a Bohlin-Levi-Civitá transformation, the same separability statement holds for the Tremblay-Turbiner-Winternitz system as well.

\section{QUANTUM MECHANICS}

Let us briefly consider the quantum mechanics of the proposed systems. The quantum versions of the
Hamiltonians (13) and (19) are obtained by replacing their kinetic term by (minus) the Laplacian and by deforming the couplings $g_{\alpha}^{2}$ to $g_{\alpha}\left(g_{\alpha}-\hbar\right)$. If the $g_{\alpha}$ are integer multiples of $\hbar$, then the system is even algebraically integrable [26].

It is then easy to deduce that the wave functions of the Calogero-oscillator and Calogero-Coulomb systems are given by

$$
\Psi_{n_{r} q}\left(x_{i}\right)=\psi_{n_{r} q}\left(x_{i} / r\right) R_{n_{r} q}(r),
$$

where $R_{n_{r} q}(r)$ are the radial wave functions of the corresponding oscillator and Coulomb problems with orbital quantum number $\ell$ having been replaced with $q$ as given by (15). For the systems on spheres and hyperboloids, the role of the radius $r$ is played by the angle $\arccos \left(x_{N+1} / r_{0}\right)$. The expressions for these radial wave functions are well known [27]. Analogously, the energy spectra of these systems are found by replacing $\ell \rightarrow q$ in the spectra of the oscillator and Coulomb systems (here we put $\hbar=1)$,

$$
\begin{aligned}
E_{\mathrm{osc}} & =\left(\sqrt{\omega^{2}+\frac{1}{4} r_{0}^{-4}}-\frac{1}{2} r_{0}^{-2}\right)\left(2 n_{r}+q+\frac{N}{2}\right) \pm \frac{1}{2} r_{0}^{-2}\left(2 n_{r}+q+\frac{1}{2} \pm \frac{1}{2}\right)\left(2 n_{r}+q+N+\frac{1}{2} \mp \frac{1}{2}\right), \\
E_{\mathrm{Coul}} & =-\frac{1}{2} \gamma^{2}\left(n_{r}+q+\frac{N-1}{2}\right)^{-2} \pm \frac{1}{2} r_{0}^{-2}\left(n_{r}+q\right)\left(n_{r}+q+n-1\right),
\end{aligned}
$$

where, as before, the upper sign corresponds to the sphere and the lower one to the two-sheet hyperboloid. In the latter case, requiring positivity restricts the principal quantum numbers. In the situation where $q$ is an integer multiple of $\hbar$, the two spectra agree with the undeformed ones, i.e. with the $S O(N)$ quadratic Casimir instead of the angular Calogero Hamiltonian, but the degeneracies are different. For the Calogero-oscillator system, the energy level is determined by the principal number $n=2 n_{r}+q$, while for the Calogero-Coulomb system, it is given by $n=n_{r}+q$, where $q$ is defined in (15). Thus, the degeneracy of the $n$th level depends on the corresponding Coxeter root system.

\section{MATRIX MODEL REDUCTION}

The superintegrability of the Calogero-Coulomb model can easily be understood in terms of a matrix model. For simplicity, we consider here the root system of $u(N) \simeq u(1) \oplus s u(N)$, which leads to the standard rational Calogero model [1], although the results can be extended to any root system.
The Hermitian matrix model for a particle in a Coulomb potential is defined by the Hamiltonian

$$
\begin{aligned}
\mathcal{H}_{\text {mat }} & =\frac{1}{2} \operatorname{tr} \mathbf{P}^{2}-\gamma\left(\operatorname{tr} \mathbf{X}^{2}\right)^{-\frac{1}{2}}=\frac{1}{2} \sum_{a} P_{a}^{2}-\frac{\gamma}{r} \quad \text { with } \\
r^{2} & =\sum_{a} X_{a}^{2} \quad \text { and } \quad a=0,1, \ldots N^{2}-1 .
\end{aligned}
$$

Here $\mathbf{P}$ and $\mathbf{X}$ denote Hermitian matrices containing $N^{2}$ momenta $P_{a}$ and coordinates $X_{a}$, respectively,

$$
\mathbf{P}=\sum_{a} P_{a} T_{a} \quad \text { and } \quad \mathbf{X}=\sum_{a} X_{a} T_{a}
$$

where we introduced a basis of $U(N)$ generators $T_{a}$ orthonormalized as $\operatorname{tr} T_{a} T_{b}=\delta_{a b}$.

It is well known that the integrals of motion of such a system are given by the angular momentum tensor and the Runge-Lenz vector,

$$
\mathbf{M}=\mathbf{X} \otimes \mathbf{P}-\mathbf{P} \otimes \mathbf{X}=\sum_{a} M_{a b} T_{a} \otimes T_{b} \quad \text { with } \quad M_{a b}=X_{a} P_{b}-X_{b} P_{a},
$$




$$
\mathbf{A}=(\mathbb{1} \otimes \operatorname{tr}) \mathbf{M}(\mathbb{1} \otimes \mathbf{P})-\frac{\gamma}{r} \mathbf{X}=\left(\operatorname{tr} \mathbf{P}^{2}-\frac{\gamma}{r}\right) \mathbf{X}-\operatorname{tr}(\mathbf{X P}) \mathbf{P}=\sum_{a} A_{a} T_{a},
$$

so that the components of the Runge-Lenz vector acquire the standard form

$$
A_{a}=\sum_{b} M_{a b} P_{b}-\frac{\gamma}{r} X_{a}=\left(\sum_{b} P_{b}^{2}-\frac{\gamma}{r}\right) X_{a}-\left(\sum_{b} X_{b} P_{b}\right) P_{a} .
$$

The matrix Hamiltonian (22) is preserved under the adjoint $S U(N)$ action. For $\gamma=0$, the corresponding reduction gives rise to the Calogero Hamiltonian [2,4,19]. The coordinate and momentum matrices are reduced, respectively, to

$$
\mathbf{X} \rightarrow\left(X_{i j}\right)=\left(x_{i} \delta_{i j}\right) \quad \text { and } \quad \mathbf{P} \rightarrow\left(P_{i j}\right)=\left(p_{i} \delta_{i j}+\mathrm{i} g \frac{1-\delta_{i j}}{x_{i}-x_{j}}\right)
$$

For nonvanishing $\gamma$, this reduction procedure leads to the Hamiltonian $\mathcal{H}_{\text {Coul }}$ of the Calogero model with a Coulomb potential (18), since the potential term in the matrix Hamiltonian (22) is also invariant with respect to the adjoint action.

From the integrals of motion (24) and (25) of the matrix model, only the $S U(N)$-invariant ones survive the reduction procedure. Such invariants can be constructed using $S U(N)$-invariant tensors. In Ref. [14] the general form of such invariants, containing angular momentum tensors only, is described using a graphical representation. They correspond to the invariants of the angular Hamiltonian $\mathcal{I}$ defined in (5). Among them are the traces of even powers of the angular momentum matrix,

$$
\mathcal{M}_{n}=(\operatorname{tr} \otimes \operatorname{tr}) \mathbf{M}^{2 n}
$$

The aforementioned graphical picture may be extended by including the Runge-Lenz vector (25). In particular, the quantities

$$
\mathcal{A}_{n}=\operatorname{tr} \mathbf{A}^{n}
$$

are $S U(N)$ invariants and, hence, yield integrals of motion in the reduced model. The matrix (25) reduces to the explicit form

$$
\mathbf{A} \rightarrow\left(2 \mathcal{H}_{\mathrm{Coul}}+\frac{\gamma}{r}\right) \mathbf{X}-r p_{r} \mathbf{P}
$$

where $\mathbf{X}$ and $\mathbf{P}$ are now defined by (27), and

$$
\mathcal{H}_{\text {Coul }}=\sum_{i} \frac{p_{i}^{2}}{2}+\sum_{i<j} \frac{g^{2}}{\left(x_{i}-x_{j}\right)^{2}}-\frac{\gamma}{r}
$$

The first integral from the series (29) is

$$
\mathcal{A}_{1}=\left(2 \mathcal{H}_{\text {Coul }}+\frac{\gamma}{r}\right) \sum_{i} x_{i}-r p_{r} \sum_{i} p_{i}
$$

The quantum system can be treated using the Dunkl operators [28]. We employ their gauged $S U(N)$ version,

$$
\nabla_{i}=\partial_{i}-\sum_{k(\neq i)} \frac{g}{x_{i}-x_{k}} s_{i k}
$$

where $s_{i k}$ permutes the $i$ th and $k$ th particle labels. Like ordinary derivatives, the operators $\nabla_{i}$ mutually commute and obey nontrivial commutation relations with the coordinates $x_{i}$,

$$
\left[\nabla_{i}, x_{j}\right]= \begin{cases}-g s_{i j} & \text { for } i \neq j \\ 1+g \sum_{k(\neq i)} s_{i k} & \text { for } i=j\end{cases}
$$

The Dunkl operators serve as a tool for the construction and study of the Calogero model and its various extensions $[4,20]$. In particular, is is easy to see that the restriction of the Hamiltonian

$$
-\left.\frac{1}{2} \nabla^{2}\right|_{\text {sym }}=\hat{\mathcal{H}}_{\text {Cal }}
$$

on totally symmetric wave functions produces the Calogero model. To construct the integrals of motion for

$$
\hat{\mathcal{H}}_{\text {Coul }}=\hat{\mathcal{H}}_{\text {Cal }}-\frac{\gamma}{r},
$$

we consider the Dunkl angular momentum operators investigated in detail in Ref. [29]:

$$
\hat{M}_{i j}=x_{i} \nabla_{j}-x_{j} \nabla_{i}
$$

Such operators commute with $\hat{\mathcal{H}}_{\text {Cal }}$ and its angular part $\hat{\mathcal{I}}$ [29]. Therefore, they commute with $\hat{\mathcal{H}}_{\text {Coul }}$ (36), too, since they depend on the angular coordinates only. Because we restrict to totally symmetric wave functions, we have to symmetrize (37) in order to get the true integrals:

$$
\hat{\mathcal{M}}_{n}=\left.\sum_{i<j}\left(\hat{M}_{i j}\right)^{2 n}\right|_{\mathrm{sym}}
$$

are the quantum analogs of the integrals (28). Note that $\hat{\mathcal{M}}_{2}$ is proportional to $\hat{\mathcal{I}}$ up to a constant.

In complete analogy with (37), one can define the quantum Runge-Lenz operator expressed via Dunkl ones as 
HAKOBYAN, LECHTENFELD, AND NERSESSIAN

$$
\hat{A}_{i}=-\frac{1}{2} \sum_{k}\left\{\hat{M}_{i k}, \nabla_{k}\right\}-\frac{\gamma x_{i}}{r} .
$$

The anticommutator in this expression guarantees its Hermiticity. However, such operators do not commute with the Hamiltonian (36). Nevertheless, their sum

$$
\begin{aligned}
\hat{\mathcal{A}}_{1} & =-\frac{1}{2} \sum_{i, k}\left\{\hat{M}_{i k}, \nabla_{k}\right\}-\frac{\gamma}{r} \sum_{i} x_{i} \\
& =\left(\sum_{i} x_{i}\right)\left(2 \hat{\mathcal{H}}_{\text {Coul }}+\frac{\gamma}{r}\right)+\left(r \partial_{r}+\frac{N-1}{2}\right) \sum_{i} \partial_{i}
\end{aligned}
$$

is a constant of motion of the system, as can be verified by direct calculation. ${ }^{2}$ The final expression in (40) is a quantum version of the classical expression (32). The quantities $\hat{\mathcal{H}}_{\text {Coul }}$ and $\hat{\mathcal{A}}_{1}$ extend the $2 N-3$ conserved quantities of the angular subsystem $\hat{\mathcal{I}}$ to the full number $2 N-1$ of conserved charges required for superintegrability.

\footnotetext{
${ }^{2}$ For $\gamma=0$ it is nothing but the first Wojciechowski integral named $F_{1}$ in Ref. [26].
}

PHYSICAL REVIEW D 90, 101701(R) (2014)

\section{CONCLUDING REMARKS}

In this paper we announce the maximal superintegrability of the generalized rational Calogero model (based on any Coxeter root system) perturbed by a Coulomb potential, on Euclidean $\mathbb{R}^{N}$ as well as on a sphere $S^{N}$ or pseudosphere $H^{N}$. The same feature holds true for a harmonic oscillator potential in place of the Coulomb one. In fact, we demonstrated that the Calogero-Coulomb and Calogero-oscillator systems at integer values of the coupling are the only isospectral deformations of the standard oscillator and Coulomb systems. It should be noted that, except for the Euclidean $A_{N-1}$ Calogero-oscillator system, these models cannot be naturally interpreted as $N$-particle systems because of nonstandard multiparticle interactions.

\section{ACKNOWLEDGMENTS}

The authors thank A. Polychronakos for encouragement at the initial stage of this investigation. This work was partially supported by the Volkswagen Foundation Grant No. I/84 496, by the Armenian State Committee of Science Grant No. SCS 13-1C114, and by Armenian National Science \& Education Fund (ANSEF) Grant No. 3501math-phys (T. H. and A. N.).
[1] F. Calogero, J. Math. Phys. (N.Y.) 10, 2191 (1969); 12, 419 (1971).

[2] M. A. Olshanetsky and A. M. Perelomov, Phys. Rep. 71, 313 (1981); 94, 313 (1983).

[3] B. Sutherland, Phys. Rev. A 4, 2019 (1971); 5, 1372 (1972).

[4] A. P. Polychronakos, J. Phys. A 39, 12793 (2006).

[5] S. Wojciechowski, Phys. Lett. 95A, 279 (1983).

[6] C. Gonera and P. Kosinski, Acta Phys. Polon. B 30, 907 (1999).

[7] C. Gonera, J. Phys. A 31, 4465 (1998).

[8] V. Ayadi and L. Feher, Phys. Lett. A 374, 1913 (2010).

[9] V. Ayadi, L. Feher, and T. F. Gorbe, J. Geom. Symmetry Phys. 27, 27 (2012).

[10] S. N. M. Ruijsenaars, Commun. Math. Phys. 115, 127 (1988).

[11] A. Khare, J. Phys. A 29, L45 (1996).

[12] P. K. Ghosh and A. Khare, J. Phys. A 32, 2129 (1999).

[13] F. Calogero, J. Phys. A 29, 6455 (1996); A. Khare, J. Phys. A 29, 6459 (1996).

[14] T. Hakobyan, O. Lechtenfeld, and A. Nersessian, Nucl. Phys. B858, 250 (2012).

[15] T. Hakobyan, D. Karakhanyan, and O. Lechtenfeld, Nucl. Phys. B886, 399 (2014); T. Hakobyan, S. Krivonos, O. Lechtenfeld, and A. Nersessian, Phys. Lett. A 374, 801 (2010); T. Hakobyan, A. Nersessian, and V. Yeghikyan, J. Phys. A 42, 205206 (2009); T. Hakobyan, O. Lechtenfeld, A. Nersessian, and A. Saghatelian, J. Phys. A 44, 055205 (2011).
[16] T. Hakobyan, O. Lechtenfeld, A. Nersessian, A. Saghatelian, and V. Yeghikyan, Phys. Lett. A 376, 679 (2012).

[17] O. Lechtenfeld, A. Nersessian, and V. Yeghikyan, Phys. Lett. A 374, 4647 (2010).

[18] M. Feigin, O. Lechtenfeld, and A. Polychronakos, J. High Energy Phys. 07 (2013) 162.

[19] D. Kazhdan, B. Kostant, and S. Sternberg, Commun. Pure Appl. Math. 31, 481 (1978).

[20] A. Polychronakos, Phys. Rev. Lett. 69, 703 (1992); L. Brink, T. Hansson, and M. Vasiliev, Phys. Lett. B 286, 109 (1992).

[21] P. W. Higgs, J. Phys. A 12, 309 (1979).

[22] E. Schrödinger, Proceedings of the Royal Irish Academy. Section A: Mathematical and Physical Sciences 46, 9 (1940).

[23] J.E. Humphreys, Reflection Groups and Coxeter Groups (Cambridge University Press, Cambridge, England, 1990).

[24] F. Tremblay, A. Turbiner, and P. Winternitz, J. Phys. A 42, 242001 (2009).

[25] S. Post and P. Winternitz, J. Phys. A 43, 222001 (2010).

[26] F. Correa, O. Lechtenfeld, and M. Plyushchay, J. High Energy Phys. 04 (2014) 151.

[27] E. G. Kalnins, W. Miller, and G. S. Pogosyan, Phys. At. Nucl. 65, 1086 (2002).

[28] C. F. Dunkl, Trans. Am. Math. Soc. 311, 167 (1989).

[29] M. Feigin and T. Hakobyan, arXiv:1409.2480. 